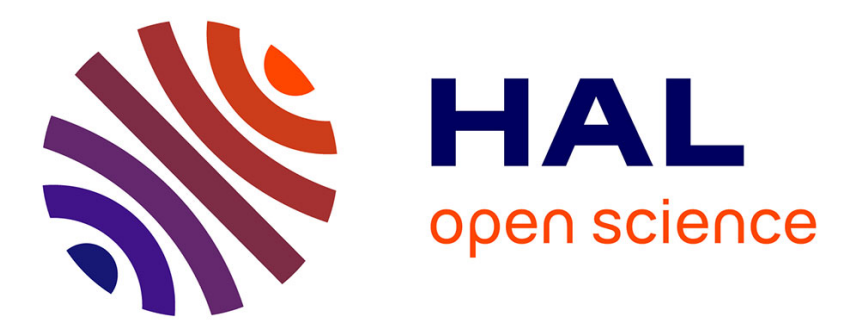

\title{
Construire l'espace sans la notion d'espace. Le cas du Salzforst (Franconie) au XIVe siècle
}

\author{
Joseph Morsel
}

\section{To cite this version:}

Joseph Morsel. Construire l'espace sans la notion d'espace. Le cas du Salzforst (Franconie) au XIVe siècle. Construire l'espace sans la notion d'espace. Le cas du Salzforst (Franconie) au XIVe siècle, 2006, Freiburg im Breisgau, Allemagne. pp.295-316. halshs-00290159

\section{HAL Id: halshs-00290159 \\ https://shs.hal.science/halshs-00290159}

Submitted on 24 Jun 2008

HAL is a multi-disciplinary open access archive for the deposit and dissemination of scientific research documents, whether they are published or not. The documents may come from teaching and research institutions in France or abroad, or from public or private research centers.
L'archive ouverte pluridisciplinaire $\mathbf{H A L}$, est destinée au dépôt et à la diffusion de documents scientifiques de niveau recherche, publiés ou non, émanant des établissements d'enseignement et de recherche français ou étrangers, des laboratoires publics ou privés. 


\title{
Construire l'espace sans la notion d'espace Le cas du Salzforst (Franconie) au XIVe siècle
}

\author{
Joseph MORSEL
}

\begin{abstract}
Vivre, c'est passer d'un espace à un autre, en essayant le plus possible de ne pas se cogner. [...] L'espace semble être, ou plus apprivoisé, ou plus inoffensif, que le temps: on rencontre partout des gens qui ont des montres, et très rarement des gens qui ont des boussoles. On a toujours besoin de savoir l'heure (et qui sait encore la déduire de la position du soleil ?) mais on ne se demande jamais où l'on est. On croit le savoir : on est chez soi, on est à son bureau, on est dans le métro, on est dans la rue. (Georges Perec, Espèces d'espace, 1974/2000)
\end{abstract}

\section{L'espace comme surface n'est qu'une évidence de notre sens commun}

Dans les années 1990, le peuple des Spinifex (Pila Nguru), un peuple aborigène d'Australie Occidentale, a demandé la restitution de ses lieux de vie ancestraux, dont il avait été chassé dans les années 1950 dans le cadre du programme nucléaire britannique. Tenu de se conformer aux règles du droit australien, qui exigeait notamment une preuve tangible du bien-fondé de cette revendication, mais étant une société sans écriture ni archives, ce peuple a alors procédé en 1998 à la réalisation de deux peintures, dues l'une aux femmes, l'autre aux hommes, et qui donnaient à voir l'espace concerné. Chacune de ces peintures constituait un réseau de doubles lignes [296] reliant de petits disques, l'espace interstitiel étant occupé par des motifs que nous appellerions « décoratifs » 1 .

Le « remplissage décoratif » de la surface entre les lignes et points renvoie au fait que l'espace que revendiquait le peuple des Spinifex n'était pas conçu comme une surface: il s'agissait d'un réseau de circulation, comme le montre bien le schéma structural qui a servi de base au travail des peintres. Mais ce que montre aussi ce schéma (qui associe à chaque disque un anthroponyme), c'est que ce qui est relié n'était pas des lieux, mais des gens : leur espace n'est pas une étendue naturelle (d'ailleurs aucun élément naturel n'apparaît dans le schéma ni les peintures), ce n'est même pas un réseau de chemins concrètement tracés, c'est un réseau de relations entre les personnes, qui sont donc conçues comme préexistantes à l'espace (selon le sens que nous donnons à ce terme), de même que leur distinction de genre (masculin/féminin)².

\footnotetext{
1. Les peintures (Women's Native Title Painting et Men's Native Title Painting) - et le schéma dont il est question plus bas - figurent dans le catalogue de l'exposition qui a été organisée autour d'eux en 2005 à l'Aboriginal Art Museum d'Utrecht: Law and Land. Art of the Spinifex People, Utrecht, 2005 (museumscahier, deel 3), p. 5-6. À la date de la présente publication, les deux peintures (mais pas le schéma) sont également visibles sur l'Internet aux adresses suivantes : http://www.spinifex.org/sml_n1.html et http://www.spinifex.org/sml_n2.html. [cf. planche suivante, qui représente le Women's Native Title Painting et le schéma]

2. Lors du Congrès de Mulhouse, Jens Schneider (Paderborn) a attiré mon attention (et je l'en remercie chaleureusement) sur le récit de voyage de Bruce Chatwin, The Songlines (1987), qui se penche précisément sur les pratiques et représentations spatiales des aborigènes australiens. Il évoque ainsi « l'existence du labyrinthe de sentiers invisibles sillonnant tout le territoire australien et connus des Européens sous le nom de songlines, "itinéraires chantés" ou "pistes des rêves", et des aborigènes sous le nom de tjurna djugurba, "empreintes des ancêtres" ou "chemins de la loi". [...] Lors de sa traversée du pays, chaque ancêtre avait laissé dans son sillage une suite de mots et de notes de musique et ces pistes de rêve formaient dans tout le pays des "voies" de communication entre les tribus les plus éloignées. » (B. CHATwin, The Songlines, Londres, 1987, trad. fr. Le chant des pistes, Paris, 1988, p. 10, 22). Les conséquences de cela sur le plan social, du point de vue de l'appropriation effective de l'espace, lui sont bien signalées par le Père Flynn (un aborigène bénédictin défroqué) : "Les hommes blancs commettaient généralement l'erreur de croire que, comme les aborigènes étaient des vagabonds, ils ne pouvaient pas avoir mis en place un système de propriété foncière. C'était une aberration. Les aborigènes, il est vrai, ne concevaient pas le territoire comme un morceau de terre délimité par des frontières, mais plutôt comme un réseau de "lignes" et de voies de communication entrecroisées. "Tous les mots que nous utilisons pour dire 'pays' sont les mêmes que les mots pour 'lignes'. [...] 'Mon pays' se définissait comme 'l'endroit dans lequel je n'ai pas à demander'.” » (ibid., p. 67).
} 

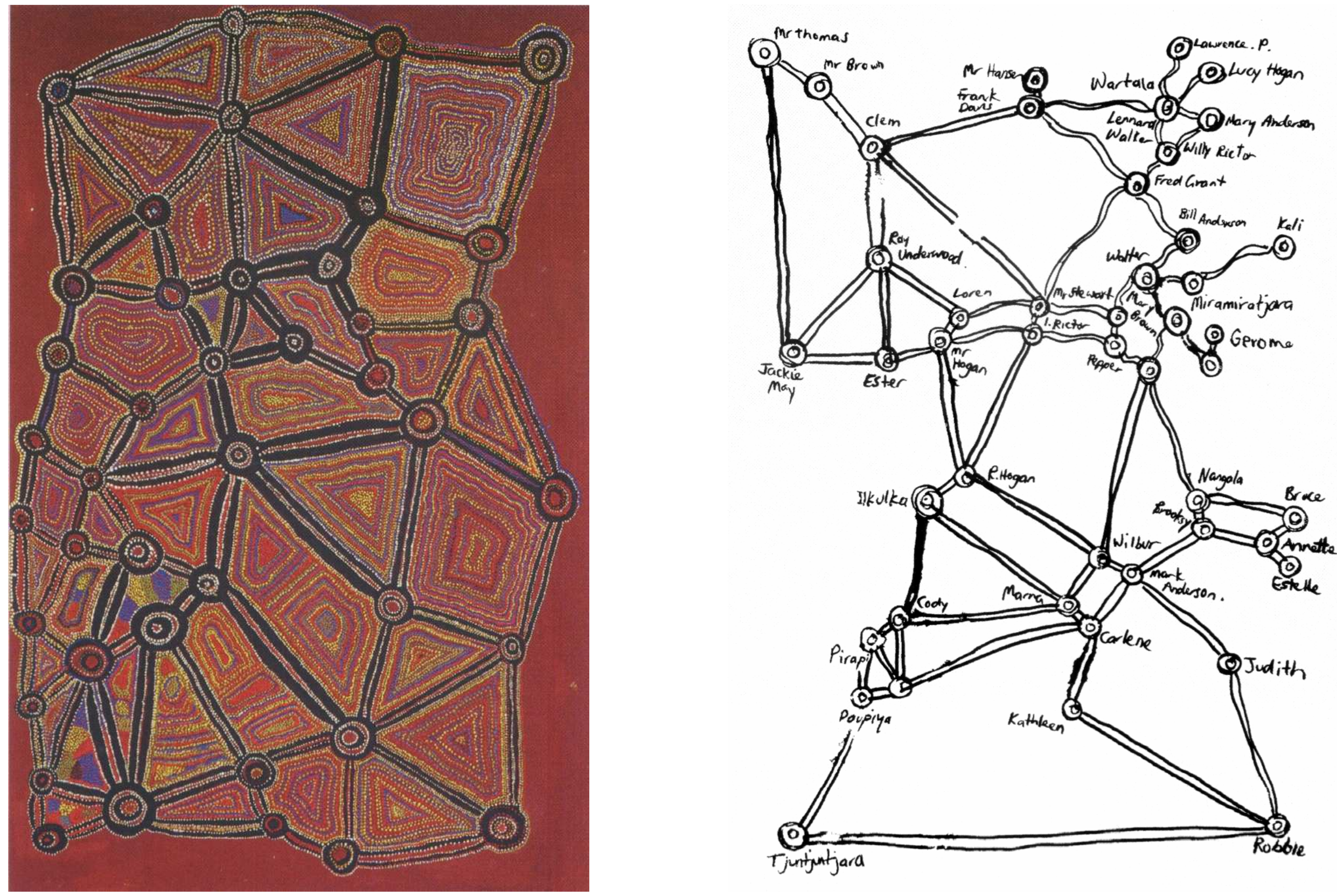
Le peuple des Spinifex a obtenu gain de cause en 2000, mais là n'est pas l'essentiel dans notre présente optique : ce qui importe ici, c'est que nous sommes mis ainsi en présence d'une forme d'appropriation de l'espace par des gens qui, contraints de se couler formellement dans le moule juridique occidental contemporain et de donner une forme tangible à des [297] pratiques ancestrales, produisent alors ce que, spontanément, nous considérerions comme une sorte de " carte », celle-ci formalisant toutefois non pas une étendue, mais des rapports sociaux.

Quel rapport y a-t-il entre le peuple des Spinifex et notre société médiévale ? Aucun. Il ne peut rien nous apprendre sur la société médiévale, ni directement, ni par transfert d'un schéma d'explication (par exemple l'espace conçu comme un réseau de liens entre des personnes). Les excès d'un "cannibalisme » pseudo-anthropologique ont été suffisamment dénoncés, de la part d'anthropologues comme d'historiens pourtant guère soupçonnables de se défier de l'anthropologie, pour qu'il soit inutile d'y revenir. Ce qu'en revanche le cas du peuple des Spinifex peut nous apprendre, c'est que nous devons nous déprendre de nos propres représentations de l'espace dès lors que nous prétendons aborder une société autre que la nôtre.

L'espace n'est en effet en rien une réalité naturelle, mais une construction sociale, parce que toute société produit son espace propre, c'est-à-dire sa manière de concevoir, de structurer et de rendre signifiants les rapports entre les éléments qui composent son environnement. Il n'est pas d'espace hors de sa définition, il n'est pas d'espace autre que social (ce qui ne signifie en aucun cas mental)3. La spatialité médiévale était donc selon toute vraisemblance différente de la nôtre, c'est-à-dire que rien ne permet de considérer a priori que notre représentation de l'espace en tant qu'étendue, en tant que surface (et en tant que volume à partir des surfaces), de manière aréolaire, valait pour le Moyen Âget. C'est d'ailleurs parce que l'idée d'un [298] espace-étendue neutre,

3. Je me permets de renvoyer ici aux observations sur ce problème (et à la bibliographie utilisable dans cette optique) que j'ai présentées dans ma contribution « Appropriation communautaire du territoire, ou appropriation territoriale de la communauté ? Observations en guise de conclusion", dans: Hypothèses 2005. Travaux de l'École doctorale d'histoire de l'Université Paris I Panthéon-Sorbonne, Paris, 2006, p. 89-104 (ici p. 91-96).

4. À l'issue de ma communication s'est amorcé un débat autour de ce postulat d'une différence fondamentale entre la spatialité médiévale et la nôtre, qui ne serait pas si différente puisque nos pratiques et représentations quotidiennes sont essentiellement organisées autour de la rue. Malgré sa relative longueur, je me permets d'insérer ici le texte de ma réponse dans la mesure où elle me paraît pouvoir clarifier certains points. 1) D'un point de vue strictement méthodologique, il est périlleux de remonter de nos pratiques de l'espace à nos représentations d'icelui : je ne dis pas que c'est impossible, comme si les deux choses évoluaient dans des sphères séparées et autonomes, mais l'articulation des deux est justement un problème clé des sciences sociales et je ne sache pas qu'on dispose d'autre chose que d'hypothèses incertaines, qui semblent cependant imposer de distinguer entre les "représentations pratiques " (articulées à une logique de l'action concrète) et les « représentations abstraites » (articulées à des discours plutôt qu'à des pratiques quotidiennes). Cela pour dire que je me garderais bien de déduire directement de nos pratiques quotidiennes de l'espace l'énoncé de nos représentations y afférentes. 2) Qui plus est, il me paraît évident que les pratiques [298] spatiales présentent des aspects très particulier puisqu'elles ne peuvent guère être que ponctuelles (la pratique des lieux : habiter, enterrer, etc.) ou linéaires (aller chez soi, au travail, faire les courses, au cinéma, en vacances, etc.) : je ne connais pas de pratiques qui soient concrètement « superficielles » (au sens propre du mot), c'està-dire qui constituent une forme d'appropriation/usage en deux dimensions (même l'agriculture fonctionne de façon linéaire, comme le montre le moindre champ labouré). Par conséquent, des pratiques ponctuelles ou linéaires ne peuvent déboucher sur une représentation aréolaire qu'à la suite d'une opération mentale particulière - raison pour laquelle j'aurais tendance à considérer que la pensée de l'espace en deux dimensions ne relève que des représentations abstraites, dont la géométrie euclidienne n'est qu'une formalisation poussée, et non pas des représentations articulées à des pratiques (nos représentations quotidiennes). 3) Surtout, dans le cas qui nous intéresse, le problème qui se pose n'est pas tant la nature culturelle de l'opération que sa nature scientifique. Car le problème n'est pas tant le rapport entre les représentations de l'espace médiévales et contemporaines qu'entre les représentations médiévales et celles des médiévistes, ce qui est un tout autre problème (même si évidemment les représentations des médiévistes entretiennent un rapport particulier avec les représentations contemporaines). En l'occurrence, il s'agit de prendre en compte le fait que les médiévistes ont été dotés par leur formation d'une pensée de l'espace en tant qu'étendue, cartographique ; et tous ont intégré la notion d'échelle, qui fait justement disparaître la rupture que j’ai soulignée plus haut entre les pratiques ponctuelles ou linéaires et les représentations bidimensionnelles. En effet, en fonction de l'échelle, un point sur la carte pourra représenter un phénomène pratiquement étendu et pratiqué sous la forme d'un enchevêtrement de circulations et d'une juxtaposition d'usages ponctuels : l'exemple type est celui d'une ville, qu'on peut réduire à un point sur la carte. Lorsque les médiévistes appréhendent la spatialité des phénomènes sociaux médiévaux (puisque telle est la raison pour laquelle nous devons, entre autres, nous préoccuper de l'espace : l'enjeu en est d'ailleurs moins de savoir ce qu'était en soi l'espace médiéval que de comprendre la spatialité spécifique des phénomènes sociaux médiévaux), ils 
antérieur au social, naturel, bref cartésien, est reconnue comme n'étant que notre sens commun (celui des médiévistes) de l'espace que le Congrès de Mulhouse a pu se donner le thème, crucial, qui est le sien cette année.

C'est ce qui explique l'absence de toute cartographie médiévale au sens propre je ne parle évidemment pas des schémas de représentation du monde, improprement appelées « cartes T-O », ni des représentations plus complexes qui en dérivent comme la fameuse mappemonde d'Ebsdorf, car aucun de ses documents n'avait pour finalité un quelconque repérage dans l'espace ou la constitution d'itinéraires ${ }^{5}$ : il s'agissait bien plutôt de [299] figurations du cadre mystique (comme on parle de corpus mysticum) du Salut, comme le manifestent clairement des figurations anglaises ${ }^{6}$ du Christ dans lesquelles son corps (corpus christi!) est remplacé par un disque T-O - ce qui me conduit à voir dans les mappa mundi qui auraient été présentes dans toutes les églises d'Angleterre non pas des cartes, mais tout simplement les Christ en croix ou quelque autre figuration christologique...

Tout le monde connaît également l'extrême variabilité des manières d'évaluer l'importance (pas la surface!) de pièces de terre, que ce soit par le recours à des référents temporels («journaux », morgen...), d'ensemencement (« sétérées »...), de rente (« soudées »...), voire à des unités de mesure dont la nature exacte nous échappe mais qui ont pour caractéristique de varier selon ce que porte le sol («arpents", «bonniers »...). On ne se trompera sans doute pas en disant que ce qui était mesuré n'était pas la surface, mais le rapport des hommes au sol7. C'est ce qui fait, à mon avis, tout l'intérêt de la forêt dans l'examen du problème de la construction de l'espace au Moyen Âge.

\section{La sylve comme moyen d'appréhender la production de l'espace médiéval}

La forêt fait en effet partie de nos évidences, de nos représentations courantes, éventuellement de nos pratiques dominicales ou saisonnières, et constitue pour nous, dans le langage commun, un paysage spécifique caractérisé par une étendue boisée (la définition courante du Robert est qu'il s'agit d'une " vaste étendue de terrain peuplée d'arbres ; ensemble de ces arbres »), à laquelle nous attachons des idées (souvent illusoires) de paysage naturel et d'air pur, parfois aussi de grand méchant loup...

[30o] Mais assimiler d'emblée la forêt à un espace boisé sous-entend deux choses : d'une part qu'il s'agit fondamentalement d'un ensemble d'arbres, c'est-à-dire de choses qui, en tant que genre ou espèce biologique, échappent très largement au temps de l'histoire. Comme toutes les évidences, la forêt est menacée par l'achronisme, et donc

rétrojettent moins une spatialité contemporaine qu'une spatialité disons «savante», abstraite. Celle-ci est certes contemporaine, mais il ne s'agit pas de ma part d'une croisade culturaliste : s’ils abordaient la spatialité médiévale à partir de leurs pratiques quotidiennes de l'espace, en tant qu'agriculteurs (ou même qu'archéologues), on pourrait peutêtre se contenter d'une posture culturaliste (ou de la dénonciation d'une telle posture). Mais ce n'est pas le cas, et c'est cette rétrojection d'une opération mentale liée à des procédures socioprofessionnelles qui m'importe. Je maintiens par conséquent que le médiéviste doit impérativement se déprendre de ses représentations spatiales lorsqu’il aborde la société médiévale, parce qu'il recourt à un instrumentaire spatial qui lui est propre et qui correspond aux représentations dominantes de notre société.

5. On observera également que les premières formes de représentation plane mimétique (c'est-à-dire censées restituer sur un plan des données observées sur le terrain en vertu d'un rapport de réduction à peu près constant) ne représentaient pas des étendues, mais des limites particulières : limites entre seigneuries, ou même limites entre terre et mer dans le cas des versions graphiques des portulans, qui ne s'intéressent qu'aux côtes.

6. Par ex. dans un psautier des environs de 1260 (Londres, British Library, Ms. Add. 28681, fol. 9r-v), reproduit dans : A.D. VON DEN BRINCKEN, 'Finis Terræ'. Die Enden der Erde und der vierte Kontinent auf mittelalterlichen Weltkarten, Hanovre, 1992 (MGH, Schriften, 36), pl. 32 et 33.

7. N'est-ce pas un phénomène de nature semblable au fait que dans notre société pourtant uniformisante en matière métrologique, nous utilisons des unités de mesure distinctes quoique aisément convertibles, le $\mathrm{m}^{2} / \mathrm{km}^{2}$ et l'are $/ \mathrm{hectare}$, selon ce que nous mesurons, l'hectare étant réservé aux étendues exploitées de manière agricole ou sylvicole? 
son historien par l'anachronisme. D'autre part, cela sous-entend que les arbres sont un caractère qui s'ajoute en plus à une surface conçue comme une sorte de page blanche, devenant une forêt quand ce sont des arbres qui poussent dessus, une vigne quand il s'agit de ceps, un champ quand il s'agit d'épis, etc. La forêt joue d'ailleurs sans doute à deux niveaux de cette métaphore de l'espace comme page blanche, puisque nous assimilons tendanciellement l'espace boisé à un espace primitif, vierge, antérieur à l'humanisation - et donc encore une fois à l'Histoire.

Par conséquent : une forêt médiévale - ou plutôt, en reprenant la proposition de Jean-Pierre Devroey ${ }^{8}$, une "sylve » (lat. silva, all. Wald) médiévale - est-elle bien la même chose qu'une sylve actuelle? Tous ceux qui ont eu l'occasion de lire le dernier livre d'Alain Guerreau et qui ne se sont pas laissés arrêter par son style sans concession (qui ne pose après tout problème que si l'on se place dans le cadre d'un refus du débat) - tous ceux, donc, qui ont eu l'occasion de réfléchir sur le cas emblématique qu'il présente de la vinea ${ }^{9}$ peuvent admettre que la question mérite au moins d'être posée, au-delà de l'apparente transparence du mot que le sens commun lui confère trop généreusement.

La critique d'A. Guerreau à propos de la vinea figure dans le chapitre sur « La sémantique historique ». Là est l'essentiel : le but d'A. Guerreau n'est pas de dire que les vignes n'existaient pas au Moyen Âge et/ou qu'il s'agissait d'une pure représentation ou d'une pure catégorie au sens kantien, que la chose n'existe donc que dans l'esprit des gens et que par conséquent, puisque les gens du Moyen Âge se faisaient une représentation très différente de la nôtre (notamment parce qu'elle était nourrie de la Bible), la vinea médiévale n'était pas vraiment une vigne. L'absence d'une telle optique substantialiste est d'ailleurs bien illustrée par la couverture du livre, où une enluminure représentant une scène de vendange est commentée: "Ceci est ou n'est pas une vigne. »

[301] Le problème n'est en effet pas seulement que la vinea soit, concrètement, très différente de notre vigne ${ }^{10}$ et qu'elle soit une représentation clé du christianisme : à s'arrêter là, on en resterait à de la lexicographie et/ou à de la philologie. Pour passer au stade de la sémantique (historique), il faut passer au niveau du sens : "L'objet vitis vitifera [et non pas vinea, qui est la désignation médiévale: A. Guerreau pose ici le problème de la réalité censée être à l'arrière-plan, le signifié] (ou aqua, ou murus...) n'est pertinent pour l'historien qu'à travers son sens. "11 C'est dire que l'objet de l'historien n'est pas la vigne, le vin, les champs, la terre, l'eau, les murs, la maison, etc. mais leur sens, nécessairement social, le sens que les hommes en société attribuent à l'objet considéré, qui n'a de sens qu'au sein de la société considérée - mais sens en fonction duquel les hommes agissent concrètement ${ }^{12}$.

Il ne s'agit donc pas de s'arrêter à la simple constatation idiographique de ce que, comme l'écrivait Queneau dans Zazie dans le métro, « les mots n'ont plus le même sens qu'autrefois », mais de s'appuyer sur l'intelligence du sens pour parvenir au système sémantique médiéval, et de là à la compréhension du système social qui lui correspond.

\footnotetext{
8. J.-P. DEVROEY, Économie rurale et société dans l'Europe franque (VIe-IXe s.), Paris, 2003, p. 29. L'auteur proposait ce terme afin d'éviter les problèmes liés à la notion médiévale de forestum et à son étymologie controversée.

9. A. GUERREAU, L'avenir d'un passé incertain. Quelle histoire du Moyen Âge au XXIe siècle ?, Paris, 2001, p. $195-202$.

10. Ibid., p. 197 : « La vinea du XI ${ }^{\mathrm{e}}$ siècle n'avait à peu près rien de commun avec une vigne actuelle : cépages différents, outils différents, apports différents, méthodes de culture totalement autres ; et pour brocher sur le tout, un produit qu'on ne peut comparer ni en quantité ni en qualité. »

11. Ibid., p. 201.

12. L'articulation du sens à la réalité sociale (et non pas à la réalité substantielle) n'est pas seulement une difficulté pour la médiévistique française: pour le cas allemand, je me permets de renvoyer à ma contribution «Soziale Kategorisierung oder historische Phantasmagorie? Erkundungen zum historischen Gebrauch von mittelalterlichen sozialen Kategorien », dans : Wirtschaft - Gesellschaft - Mentalitäten im Mittelalter. Festschrift zum 75 . Geburtstag von Rolf Sprandel, H.-P. BAUM, R. LENG et J. SCHNEIDER dir., Stuttgart, 2006, p. 211-237.
} 
D'ailleurs, comment interpréter l'absence de l'entrée silva dans le dictionnaire de J.F. Niermeyer sinon comme le signe que le mot est censé être transparent? Cette transparence postulée repose sur (et reproduit) l'idée que nous savons fondamentalement ce qu'est une sylve et que seuls peuvent en varier, dans le temps et dans l'espace, la forme (extension et composition) et les usages pratiques.

Une sylve médiévale est-elle donc bien la même chose qu'une forêt actuelle, c'està-dire un espace boisé plus ou moins étendu ? Comme pour la vinea, on pourrait montrer que, malgré la continuité biologique des espèces, il s'agissait d'un système écologique radicalement différent de celui qu'impliquent aujourd'hui nos pratiques de sélection des espèces, de plantation, d'écobuage, nos pluies acides, etc. Bref, c'est un autre système écologique parce que notre rapport collectif, social, à la sylve a changé [302] - changement dont la disparition des mots courants médiévaux (silva, saltus et leurs dérivés romans) est sans doute un signe fort ${ }^{13}$.

Pourtant, si l'on se contente d'examiner ce qui était concrètement désigné par les mots silva (ou selve, ou wald...), on y trouvera évidemment des arbres et aussi des bêtes sauvages, censés être, on l'a dit, des référents anhistoriques - et l'on en déduira donc que c'était fondamentalement la même chose. Or rien n'est moins sûr, parce que rien ne permet de considérer que la mention d'arbres ou de bêtes sauvages allait de soi, comme si elle ne servait qu'à décrire/illustrer ce qu'était une forêt. Le sens des arbres, tout comme celui des bêtes sauvages, n'était pas fixe, substantialiste, descriptif, mais propre non seulement à cette société mais aussi à ce champ lexical précis : il est évident que le sens de l'arbre présentera des inflexions significatives (mais pas nécessairement exclusives) selon qu'on se trouvera dans un commentaire sur l'arbre de Jessé ou la croix du Christ, dans un discours sur la filiation aristocratique ou dans une ordonnance sur la coupe du bois. La mention de ces prétendues réalités servait donc à dire quelque chose sur la forêt, et surtout à la qualifier en tant que forêt.

Mais on pourrait également montrer, à partir d'une étude sémantique des termes silva, saltus, nemus, etc. dans la Vulgate, les textes patristiques et les gloses théologiques, que la sylve occupait une place tout à fait particulière dans les représentations dominantes médiévales, très différentes de nos représentations actuelles. Pour des raisons de place, mais aussi parce que cela constituerait une recherche en soi, je ne m'y suis pas vraiment livré et me suis contenté de relever les usages qu'en font la Vulgate et saint Augustin ${ }^{14}$.

Contrairement à la vinea, la silva est très peu présente dans la Vulgate : une cinquantaine d'occurrences, presque exclusivement dans l'Ancien [303] Testament, dont plus d'un quart dans le seul quatrième livre (apocryphe) d'Esdras, les autres occurrences étant le plus souvent des hapax dans les livres où elles apparaissent. Silva y est essentiellement associée à la mention d'animaux (gazelles, bêtes féroces, sangliers et surtout lions) et de lignum, en quoi elle est opposée à l'ensemble ager/campus/vinea. Moins fréquemment, l'association est faite avec mons ou desertus. Mais chez Augustin,

\footnotetext{
13. Le mot saltus n'a laissé aucun héritage en français : sa forme romane sault ne subsiste plus que dans des toponymes (W. VON WARTBURG, Französisches Etymologisches Wörterbuch, t. 11, Bâle, 1965, p. 125), dont la très grande fréquence (jusqu'au Québec !) montre à quel point le terme était commun. Et déjà au XVI ${ }^{\mathrm{e}}$ siècle, le mot roman selve était considéré comme un vieux mot au regard de celui de forest (E. HuGUET, Dictionnaire de la langue française du seizième siècle, t. 6, Paris, 1965, p. 750).

14. A également été recherché saltus, présent une quarantaine de fois dans le seul Ancien Testament (près d'un tiers des occurrences dans le seul livre d'Isaïe), jamais dans le Nouveau Testament; le mot est quasiment absent chez Augustin (2 à 4 occurrences en fonction de l'attribution des textes, et la plupart sont de simples citations de la Bible, à l'exception d'un sermon d'Augustin, où le Christ est évoqué par la métaphore ex semine sancti Spiritus propagata in saltu virginali primitiva vitis (PL 47, col. 1144). Quant à forestum, il s'agit d'une invention médiévale ; le terme est donc logiquement absent de la Vulgate et des textes d'Augustin, tout comme des 86 premiers tomes de la Patrologie latine. Le mot n'y apparaît que vers 650 , dans des préceptes royaux mérovingiens.
} 
chez qui le terme reste également peu fréquent ${ }^{15}$, c'est l'association aux animaux sauvages qui est prédominante par rapport au bois, lorsque la sylve est évoquée en tant que telle.

Car très souvent aussi, la mention est purement métaphorique, pour désigner un ensemble embrouillé, épineux, obscur, où l'on se perd aisément, proche de notre métaphore du «maquis ». De façon encore plus orientée, la sylve est associée à la présence du mal, sous la forme des péchés et crimes, des démons, des païens ou juifs (dont les bêtes sauvages et, secondairement, les arbres, sont des figures). C'est donc un lieu fondamentalement stérile, qui contraste évidemment avec nos propres représentations de la forêt comme principale source de biomasse... ${ }^{16}$, et qui s'oppose donc logiquement aux agri dominici, vinee et tout simplement à l'ecclesia ${ }^{17}$.

On est donc bien ici aux origines de la « forêt obscure » de Dante - et à l'opposé absolu du Génie du christianisme. Mais cette forêt obscure détermine aussi le statut particulier de la forêt dans les romans de chevalerie, en tant que pôle complémentaire et opposé, cette fois, à la cour et à château (qui constituent l'équivalent structural de l'église dans cette littérature), et lieu de [304] l'aventure où le chevalier doit affronter les diverses figures du Mal pour devenir le chevalier (chrétien) accompli ${ }^{18}$.

Mais surtout, au-delà de ces données écologiques et idéologiques, on peut observer que la sylve se caractérise également par une spatialité particulière, spécifiquement construite et qui fait exister la sylve en tant que telle - bref, que la sylve médiévale ne se définirait pas plus par rapport à l'espace (au sens où nous l'entendons aujourd'hui) que par rapport au boisement ${ }^{19}$. Il ne s'agit pas là d'une pure provocation, mais de la conséquence logique des observations générales faites au début de cet article. Et il me semble que le cas du Salzforst permette à la fois d'appréhender la construction de la spatialité sylvestre et donc de progresser dans l'appréhension de spatialités autres.

\footnotetext{
15. 116 occurrences probables dans la Patrologie latine, avec le degré d'incertitude lié à la tradition des œuvres d'Augustin. Le doublement des occurrences entre la Vulgate et Augustin ne doit pas tromper : la première ne compte que 658000 mots environ, soit près de six fois moins que l'œuvre d'Augustin dans la Patrologie latine (environ 4853 ooo mots).

16. Le fait que la « sylve » puisse être une source d'arbres n'est pas alors un facteur permettant de considérer que la forêt est un lieu fécond: en tant que «lieu d'extraction de bois », la sylve n'est guère différente d'une carrière. Car la « pousse » des plantes n'est pas un signe de fécondité, le processus de pousse n'est pas considéré en soi, en tant que processus naturel, mais uniquement par rapport au travail agricole : il faut qu'il y ait travail humain pour qu'il y ait fécondité... D'ailleurs, dans les Weistümer ici utilisés, on observe que les arbres ne sont évoqués que de manière générique, typologique, selon leur usage ou leur essence, et non pas en tant qu'éléments du paysage : ils sont donc du bois plus que des arbres.

17. Il n'y a pas opposition avec la mention du sermon d'Augustin (cf. note 14), car le saltus dont il y est question est un saltus virginal, c'est-à-dire spirituel, donc fécond. L'examen du sens de saltus dans la Vulgate montre que le mot semble plus ambivalent que silva : tout autant associé que silva aux arbres et aux bêtes sauvages (ourses, lions, bêtes féroces), également associé à montes et à desertum, sa destruction par le feu est classiquement présentée comme une métaphore du châtiment divin ; mais en même temps le saltus figure comme métaphore d'Israël. L'absence du mot dans le Nouveau Testament connote clairement le saltus avec la période prophétique.

18. Sur la sylve dans les romans de chevalerie, on se reportera à l'article classique de J. LE GofF et P. VIDAL-NAQUET, "Lévi-Strauss en Brocéliande. Esquisse pour une analyse d'un roman courtois ", Critique, 325 (1974), p. 543-571 (complété et rééd. dans J. LE GoFF, Un autre Moyen Âge, Paris, 1999, p. 581-634), et surtout à A. GUERREAU-JALABERT, "L'essart comme figure de la subversion de l'ordre spatial dans les romans arthuriens », dans Campagnes médiévales: l'homme et son espace. Études offertes à Robert Fossier, É. MORNET dir., Paris, 1995, p. 59-71.

19. Dans le Weistum présenté dans la seconde moitié de cet article, la clause 14 montre bien que des champs abandonnés font partie de la forêt, alors que l'on ne peut y trouver des arbres : au mieux, au bout de quelques années, des arbustes de reforestation (bouleaux, noisetiers) et des buissons. Forêt ne rime donc pas avec futaie : même les clairières font partie de la forêt, qui est donc bien plutôt un espace qu'un paysage.
} 


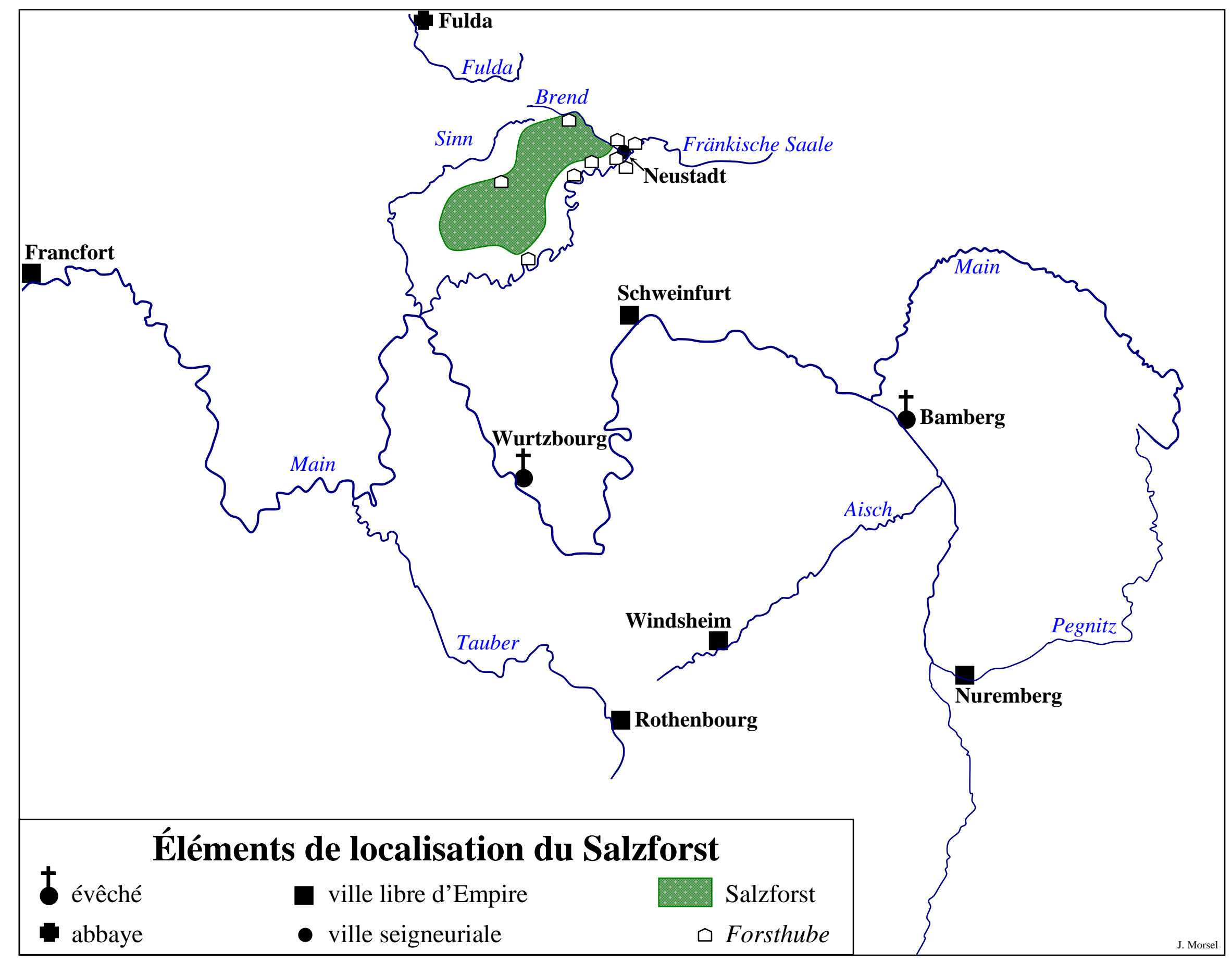




\section{Le Salzforst et ses Weistümer}

Le Salzforst est un massif boisé au nord de l'évêché de Wurtzbourg (Franconie), occupant l'interfluve entre Fränkische Saale, Sinn et Brend, c'est-à-dire aux confins des terres des abbés de Fulda (aujourd'hui en Hesse) et des comtes de Henneberg (aujourd'hui en Thuringe). Il constitue la partie méridionale d'un ensemble appelé silve Buchonia, remontant vers le nord au-delà de Fulda, et dont le nom trahit la prédominance des hêtres dans son boisement. Le nom de Salzforst provient du château de Salz, situé en face de la ville de Neustadt (aujourd'hui Bad N. an der Saale) et donné par Otton III à l'évêque en l'an 1000 en même temps que toutes les silvæ et villæ ressortissant au château ${ }^{20}$.

[305] On conserve pour le Salzforst une série de documents en général désignés comme des Weistümer ("aveux de droits») ${ }^{21}$, énonçant les droits de l'évêque de Wurtzbourg, à partir de $1326^{22}$. Avec ce type de document, on n'est donc ni dans le domaine littéraire, ni dans le domaine théologique, mais dans le domaine normatif, avec une nette référence au "droit ». Et de fait, l'analyse lexicométrique du texte fait apparaître au premier plan (mis à part les articles, conjonctions et particules, et après lemmatisation et désagglutination) les termes sollen (« devoir»), recht (« droit»), wald (« sylve »), forst (« forêt ») et haben (ici « posséder »).

On pourrait renforcer l'idée d'un document « réaliste» (par opposition au littéraire et au théologique, et en supposant une adéquation du normatif et du réel) en le replaçant dans le contexte habituellement retenu pour ce qui est de l'enjeu social de la forêt à la fin du XIII et au début du XIV siècle, à savoir celui d'une pression accrue sur la forêt, d'une concurrence entre seigneurs et entre seigneurs et villageois pour l'accès à la forêt, de mises en défens et de création d'institutions de surveillance et de gestion seigneuriale des forêts. Les Weistümer sur le Salzforst semblent parfaitement s'intégrer à ce schéma, avec leur série de clauses affirmant les droits épiscopaux sur la forêt et la définition précise des usages quant à la chasse, à la prise de bois et à la pâture.

Tous ces éléments pourraient conduire à considérer que l'objet des Weistümer en question est la transformation d'un wald en forst (d'une " sylve » en " forêt »), c'est-àdire en un ensemble séparé et surveillé, soumis à une administration strictement forestière ${ }^{23}$ et placé hors du droit commun ${ }^{24}$; d'ailleurs, même la procédure qui donne naissance au Weistum [306] diverge de ce qu'on observe habituellement ${ }^{25}$. Toutefois,

20. Die Urkunden Otto des III., Th. SicKel éd., Hanovre, 1893 (MGH, Die Urkunden der deutschen Könige und Kaiser 2, 2), $n^{\circ} 361$. [Tous les lieux mentionnés figurent sur la planche supplémentaire qui précède]

${ }^{21}$. Sur ce type de document et ses implications sociales, je me permets de renvoyer à ma contribution "Le prélèvement seigneurial est-il soluble dans les Weistümer? Appréhensions franconiennes (1200-1400)», dans Pour une anthropologie du prélèvement seigneurial dans les campagnes de l'Occident médiéval (XI ${ }^{e-X I V e}$ siècles). Réalités et représentations paysannes, M. BOURIN, P. MARTINEZ SOPENA dir., Paris, 2004, p. 155-210.

22. Édités par la Königliche Bayerische Akademie der Wissenschaften dans les Monumenta Boica, t. 39, Munich, 1866, $\mathrm{n}^{\circ}$ CXXXIX (1326); t. 42, Munich, 1874, $\mathrm{n}^{\circ}$ XVII (1353) et Nachtrag $\mathrm{n}^{\circ}$ XX (1368) ; t. 43, Munich, 1876, $\mathrm{n}^{\circ}$ LXXXVI (1377). Les passages cités dans la suite de cette contribution sont systématiquement tirés de la version de 1326, éditée ici en annexe, mais leur teneur se retrouve dans toutes les versions suivantes, en dépit de variations graphiques, syntaxiques et de position dans le texte.

23. Les Weistümer en question placent le Salzforst sous l'autorité du bailli (vogt) épiscopal de Neustadt, qui a sous ses ordres le maître-forestier (forstmeister) du Salzforst, lequel domine les divers [gardes] forestiers (forster), dotés chacun d'un ressort particulier (amt).

24. C'est ce que montrent bien les peines prévues à l'encontre des braconniers, proprement liées au délit (cynégétique) perpétré : [6] «On doit brûler le filet sur le dos du poseur de filets, item couper le pouce droit d'un poseur de collets, item couper le pied droit d'un poseur de pièges ».

25. Habituellement, ce sont les villageois qui énoncent les droits du seigneur : cf. J. MORSEL, «Le prélèvement... », op. cit., ainsi que " Quand faire dire, c'est dire. Le seigneur, le village et la Weisung en Franconie du XIII ${ }^{\mathrm{e}}$ au XVe siècle », dans Information et société en Occident à la fin du Moyen Âge. Actes du colloque international tenu à l'Université du Québec à Montréal et à l'Université d'Ottawa (9-11 mai 2002), C. BOUDREAU et al. dir., Paris, 2004, p. 309-326. Mais dans le cas des Weistümer ici envisagés, ce sont tous les maîtres-forestiers épiscopaux qui ont constitué l'assise forestière (forstding) au cours de laquelle a eu lieu cette énumération. 
cette construction forestière ne saurait se réduire à une simple procédure juridique visant à requalifier un espace par imposition d'une norme sous forme de clauses prescriptives ou prohibitives qui n'entraîneraient que de nouvelles pratiques spatiales sur fond d'espace immuable.

La place manque pour s'étendre ici sur la normativité médiévale, dont je rappellerai simplement, à la suite des travaux de Claude Gauvard sur le Parlement, de Martine Grinberg sur les coutumes ou encore de Julien Demade sur les arrérages ${ }^{26}$, qu'elle vise moins à imposer le respect d'une norme absolue et extérieure qu'à définir un cadre de négociation rationnelle. Surtout, une lecture juridiste pose assez rapidement des problèmes, dans la mesure où, au milieu des mesures évoquées, apparaissent des clauses "étranges », par leur contenu ${ }^{27}$ mais aussi par leur forme ${ }^{28}$. Ces mesures sont maintenues dans toutes les versions successives examinées, avec quelques adaptations qui montrent certainement qu'elles ne sont pas seulement un tic d'écriture.

À moins de considérer, comme on l'a longtemps fait, qu'il s'agit là d'une nouvelle manifestation du caractère irrationnel en même temps que grotesque des médiévaux, ou à l'inverse, surtout au sujet des Weistümer, qu'il s'agit d'un signe de leur fraîcheur et donc du caractère original des documents - le résultat dans les deux cas étant que ces mesures étaient laissées de côté -, celles-ci doivent être pleinement intégrées à l'analyse des [307] documents en question. Ce faisant, elles montrent combien la définition forestière du Salzforst passe par la définition d'une spatialité spécifique.

\section{Une spatialité sans étendue}

L'examen du vocabulaire employé permet d'observer l'absence de toute notion renvoyant globalement au Salzforst en tant qu'espace : il n'y a aucun terme évoquant l'idée d'une étendue, d'une aire, d'un espace ${ }^{29}$; au mieux, on est " dans la sylve », ou " dans le Salzforst », sans que rien ne soit dit de sa nature ou de sa forme spatiales. Les seules parties du Salzforst qui fassent l'objet de mentions explicites sont d'une part les chemins (wege) qui le parcourent et d'autre part, surtout, sa périphérie (ingange, uzgange, banmüle, zil). Inversement, le « contenu », l'étendue entre les chemins ou le cœur de la sylve (par opposition à la périphérie), n'est jamais évoqué.

Il s'agit donc à première vue d'un espace qui se définirait plutôt par rapport à sa périphérie ${ }^{30}$. Mais il convient d'aller plus loin, en observant que la définition par rapport à l'extérieur ne se réalise pas du tout par délimitation, comme on le fait par exemple pour un finage, par circumambulation et/ou bornage, ou comme on aurait pu

26. Cl. GAUVARD, «Le jugement entre norme et pratique : le cas de la France du Nord à la fin du Moyen Âge », dans Norm und Praxis im Alltag des Mittelalters und der Frühen Neuzeit, G. JARITZ dir., Vienne, 1997, p. 27-38, et surtout «Les juges jugent-ils ? Les peines prononcées par le Parlement criminel, v. 1380-v.1435», dans Penser le pouvoir au Moyen Âge, VIIIe-XVe siècles. Études d'histoire et de littérature offertes à Françoise Autrand, D. BOUTET, J. VERGER dir., Paris, 2000, p. 69-87; M. GRINBERG, Écrire les coutumes. Les droits seigneuriaux en France, Paris, 2006; J. DEMADE, « La fonction de l'endettement et de la justice dans le rapport seigneurial, ou la grâce comme contrainte (Franconie, XVe s.) », dans La dette et le juge. Juridiction gracieuse et juridiction contentieuse du XIII e au XVe siècle (France, Italie, Espagne, Angleterre, Empire), J. Claustre dir., Paris, 2006, p. 69-119.

27. Clause 4 ( « Il a été aussi déclaré sous la foi du serment que dans la dite forêt, personne ne peut chasser à tir s’il n'a un cheval blanc et un braque blanc aux oreilles dressées, un arc en bois d'if avec une corde de soie, une flèche à fût [de plume] d'autruche et pointe d'or, empennée de plumes d'aigle »).

${ }^{28}$. Quatrain rimé au milieu du texte (clause 5 , dont le sens sera abordé plus loin).

29. Rappelons que les termes médiévaux qui ont donné le moderne « espace » (spatium, rûm/raum) ne signifiaient pas " espace » proprement dit, mais " intervalle », « distance », " déplacement », et que leur sens était également temporel (spatium temporis, etc.).

30. Rappelons que dans des documents du haut Moyen Âge, comme le polyptyque d'Irminon, l'importance d'une forêt est déterminée soit par son pourtour, soit par le nombre de porcs qu'on peut y faire paitre, et non par sa surface (au contraire des champs, prés et vignes, mesurés en bonniers ou arpents). 
le faire, pour une forêt, par marquage des arbres voire encerclement par un mur (cas du parc de Vincennes), mais par des pratiques spatiales spécifiques.

D'une part, en effet, cette périphérie est définie par l'implantation de tenures (huben) particulières. Les Weistümer évoquent ainsi l'existence de deux types de tenures spécifiques: les tenures forestières (forsthuben) et les tenures de lisière (zilhuben). Les tenures forestières sont tenues en fief par les forestiers de l'évêque, et les registres féodaux montrent que de ces tenures relevaient un courtil et des prés, champs et vignes. J'ai pu localiser une dizaine de ces tenures : elles se situent toutes à la périphérie immédiate du Salzforst, dont elles soulignent l'extension contemporaine ${ }^{31}$.

[308] Il en allait très certainement de même pour les zilhuben, dont le nom signale très probablement le caractère limitrophe (zil = "limite, borne, terme »), mais on ne connaît rien d'elles, probablement parce qu'elles étaient concédées sans corrélation avec un office, c'est-à-dire à des dépendants "normaux ». Mais à la différence des tenures forestières, qui sont installées dans les agglomérations périphériques, le nom même de ces tenures suggère qu'elles étaient installées directement à la périphérie de la «sylve ». Là où n'existaient pas des éléments inamovibles (cours d'eau, arbre remarquable - précisément appelé zilbaum -, routes), ces zilhuben devaient constituer le signe visible et mémorable de la limite de la forêt, peut-être là où les chemins (principaux ?) entraient dans la forêt ${ }^{32}$.

Ces deux types de tenures avaient cependant en commun de procurer à leurs détenteurs des avantages particuliers (franchise fiscale ou d'accès au bois d'œuvre). Ceci avait deux conséquences : leurs détenteurs devaient être intéressés à les conserver, donc à ne pas en être privés par leur négligence. Mais en même temps, cela signalait le caractère « extra-ordinaire » du régime forestier - puisque dans la société médiévale, le mode d'accès aux biens (appropriation, circulation, destruction) n'est jamais réductible à une simple pratique matérielle: ce mode d'accès aux biens qualifie et classe socialement les biens et les personnes.

Ce caractère "extra-ordinaire " se manifeste également bien - deuxième pratique spatiale - dans la manière dont est évaluée la profondeur de l'orée du Salzforst, là où le gibier doit aller en paix. En tant que zone limite entre le « plain » et le «bosc », l'orée faisait l'objet de définitions particulières [309] dans les divers règlements forestiers ${ }^{33}$. Dans le Salzforst, la profondeur de cet espace est définie d'une manière inhabituelle (clause 5), puisque c'est par l'addition d'un son de cor, d'un aboiement, d'un jet de marteau et d'un pet de valet. Une telle addition a un statut textuel particulier : elle est présentée sous forme de poème rimé (hornes geschelle /

\footnotetext{
31. Altenberg, Brend (aujourd'hui disparu, à proximité d’Unterweißenbrunn), Ebersbach, Elfershausen, Hohn, Lebenhan, Rode, Salz, Schondra, ainsi que Bruwarts et Huthen (lieux non localisés) : Staatsarchiv Würzburg, Stb. 728, f ${ }^{\circ}$ 209r$240 \mathrm{r}$; lf. 11, fo $27 \mathrm{v}, 41 \mathrm{v}, 77 \mathrm{r}, 78 \mathrm{r}, 82 \mathrm{r}, 84 \mathrm{v}-86 \mathrm{v}$; lf. 21 , f ${ }^{\circ} 31 \mathrm{v}, 68 \mathrm{r}, 74 \mathrm{r}, 116 \mathrm{r}$, 119r ; lf. 29, fo $82 \mathrm{v}, 98 \mathrm{r}, 102 \mathrm{v}, 153 \mathrm{r}$; lf. 30 , $\mathrm{f}^{\mathrm{o}}$ 2v, 85r ; lf. 31, f $155 \mathrm{v}$; Das älteste Lehnbuch des Hochstifts Würzburg 1303-1345, H. HoFFMANN éd., Wurtzbourg, 1972-73, p. 66. [Les Forsthuben localisées figurent sur la planche supplémentaire qui précède]

32. Consécutivement à ma communication, Wolfgang Haubrichs (Sarrebruck) m'a fourni (et je l'en remercie chaleureusement) à propos de zil et de zilbaum les données onomastiques rassemblées dans le cadre du Lexikon der Straßenbezeichnungen auquel il collabore ainsi que les données provenant du Südhessisches Flurnamenbuch, éd. H. RAMGE, Darmstadt, 2002, p. 1008-1009. Il ressort de tout cela que, étant donnée son évolution morphologique, zilbaum n'a très certainement rien à voir avec zil (avec un $i$ court, signifiant « limite, etc. »), mais plutôt avec zîl (avec un $i$ long, qui donne plus tard Zeil-), signifiant « haie » au sens de «buisson (d'épine) servant de barrière », si bien que zilbaum reste concevable comme « arbre d'une haie bordière ». Pour zilhube, l'étymon est inconnu (zil ou zîl ?), mais W. Haubrichs suggère également qu'il ait pu s'agit du féminin zîl, zîle (la forme « haie » étant masculine ou neutre), qui signifie « rangée, ligne, ruelle »; aucune de ces étymologies n'exclut la dimension bordière, mais la dernière ajouterait en plus la possibilité d'une localisation au bord d'un chemin, par exemple à l'endroit où un chemin entre dans la sylve. 33. Dans la forêt de Breteuil, en Normandie, la profondeur de cet espace particulier où s'exerçaient encore certains droits était déterminée par la visio plani, c'est-à-dire tant que l'on voyait encore le jour, ou plutôt le " plain ", à travers l'ensemble des fûts: M. ARNOUX, «Perception et exploitation d'un espace forestier : la forêt de Breteuil (XI'-XV siècles) », Médiévales, 18 (1990), p. 26.
} 
hundes gebelle / hamers wurf / schalkes furtz), qui fait se succéder des phénomènes de portée spatiale décroissante.

On aurait certainement tort de ne voir dans cette clause soit que pratique triviale et grotesque, soit qu'absurdité et folklore. Ce qu'elle montre, par la forme comme par le fond, c'est l'existence d'un écart, ici entre l'évaluation de la profondeur de l'orée et les autres modes médiévaux d'évaluation de la distance - et donc la particularité de l'objet mesuré, si l'on considère, à la suite des travaux initiés par Alain Desrosières ${ }^{34}$, que le mode de mesure contribue à la construction sociale de l'objet mesuré. Par rapport aux modes médiévaux d'évaluation de la distance, fondés soit sur des modules particuliers comme la perche, la lieue (meile, ici présente dans bannmeile) etc., variables d'un endroit à l'autre, soit sur des durées (aller-retour entre tel et tel point, journée de cheval, etc.), ce mode de calcul de la profondeur de l'orée apparait en effet comme tout à fait particulier.

D'une part, la distance y est mesurée essentiellement par le son (le cas du marteau étant peu clair35), ce qui correspond par ailleurs à l'une [310] des caractéristiques premières de la chasse dans les romans de chevalerie comme dans les traités de vénerie : le bruit (cor, chiens, cris des rabatteurs, cris des chasseurs...) ${ }^{36}$. D’autre part, étant donnée la particularité de ce mode de mesure (que met bien en valeur la forme textuelle, sous forme d'un quatrain rimé), cela constitue la forêt comme un espace d'une autre nature, où les critères courants ne valent plus.

\section{La production d'un espace à part}

Ceci est d'autant plus net qu'il en va de même de la mesure du temps forestier. Alors que partout en Occident, le temps annuel est mesuré par l'Église (cycle pascal, de Noël,

\footnotetext{
34. A. DESROSIÈRES, La politique des grands nombres. Histoire de la raison statistique, Paris, 1993.

35. Ou plutôt: c'est la présence du marteau qui fait apparaître les trois autres phénomènes comme des modes de qualification spatiale de la sylve, en en « sonorisant » la mesure. Le recours pratique au son de cor, à l'aboiement et au pet comme moyens de mesure d'une distance spécifique paraît peu évident; inversement, le lancer de marteau se rencontre à plusieurs autres reprises dans des opérations de mesure d'un glacis périphérique forestier : Weisthümer, t. 1, J. Grimm éd., Göttingen, 1840 (réimp. Berlin, 1957), p. 534, 638 ; la même chose est faite, également pour déterminer l'espace de poursuite du gibier, en jetant une hache : ibid., p. 582. Le quatrain rimé contribue ainsi à mêler une mesure effective (forestière) à des mesures qui paraissent bien moins pratiques. Si l'on admet qu'il ne s'agit pas d'une irrationalité, on pourra alors convenir que cette mixtion avait comme effet de définir la partie mesurée non par sa (seule) profondeur, mais aussi par sa nature, comme lieu de chasse. Mais alors que vient faire le pet ici, mis à part sa dimension auditive ? La " pétologie » médiévale ne me semble pas avoir retenu jusqu'alors l'attention des historiens, ce qui ne facilite guère la tâche. Toutefois, on pourra se rappeler ici le châtiment infligé à l'épouse adultère de saint Gengoul, dont les paroles sont remplacées par des pets (buccaux), qu'Anita Guerreau-Jalabert qualifie ainsi d'« antiparole» ("Saint Gengoul dans le monde : l'opposition de la cupiditas et de la caritas », dans Guerriers et moines. Conversion et sainteté aristocratiques dans l'Occident médiéval (IX'e-XIIe siècles), M. LAUWERS dir., Antibes, 2002, p. 279). Dans la culture cléricale, le pet vaut comme l'inverse de l'exsufflation de l'âme (ce qui apparaît bien, par exemple, sur un vitrail haut-allemand du début du Xvie siècle conservé à l'Art Institute de Chicago, sur lequel un démon recueille l'âme de Judas sortant de ses intestins). En tant que forme de profération diabolique, le pet serait donc tout à fait à sa place dans la sylve telle que la concevait au moins la culture dominante. Mais on doit aussi observer combien ce dernier vers répond au premier : deux formes de souffle bruyant, l'un à grande portée et socialement valorisé dans l'aristocratie, l'autre à plus faible portée et socialement dévalorisé (du moins aux yeux de la culture cléricale - mais le maître du Salzforst du Weistum est un évêque). Dans tous les cas, le sonore est bien là, et l'articulation avec la forêt et/ou la chasse plausible.

36. Le caractère acoustique de la chasse a été souligné à diverses reprises : A. GUERREAU, « Chasse », dans : Dictionnaire raisonné de l’Occident médiéval, J. LE GOFF, J.-C. SCHMITT dir., Paris, 1999, p. 166-178; J. FRIED, « Kaiser Friedrich II. als Jäger », dans : Jagd und höfische Kultur im Mittelalter, W. RöSENER dir., Göttingen, 1997, p. 149-166 (ici p. 162); J. MORSEL, "Jagd und Raum. Überlegungen über den sozialen Sinn der Jagdpraxis am Beispiel des spätmittelalterlichen Franken », ibid., p. 255-287 (ici p. 283). Il n'échappera à personne que le son était précisément la meilleure forme de repérage et de présence dans les endroits où la vue ne pouvait porter (cf. ce qui a été dit supra, note 33, de la visio plani, comme si l'auditio entrait en action dès lors que la visio cessait... C'est exactement ce qui se produit

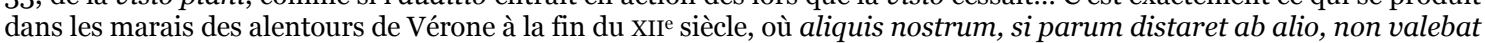
se illi coniungere neque se videre seu audire, nisi se alterutrum alta voce vocarent (A. CASTAGNETTI, " Primi aspetti di politica annonaria nell'Italia comunale : la bonifica della palus comunis Verone (1194-1199) », Studi medievali, 1974, p. 416).
} 
sanctoral), on a affaire ici à un autre mode de définition des périodes dans la forêt, par rapport au rythme de vie du cerf: le «refait» (roete, moment où les bois recommencent à pousser et sont couverts d'une peau très riche en vaisseaux sanguins, en mars/avril), la " cervaison » (veiste, moment où le cerf est le plus gras, en été) et le « rut » (bru enft, 15 septembre/début octobre).

Dans la forêt, le «temps de l'Église » (J. Le Goff) n'a donc pas cours, ce qui n'est pas nécessairement à considérer comme une remise en cause de la primauté de l'Église : rappelons que le seigneur ici concerné est l'évêque de Wurtzbourg. On devrait plutôt sans doute opposer le temps de l'Église [311] en tant que temps des hommes (chrétiens) au temps de la forêt comme temps des bêtes. Par les modes de mesure de la distance ou du temps qui lui sont appliqués, la sylve est ainsi constituée comme une unité (nous dirions " un espace ») à part du monde humain, cultivé, chrétien - ce qui correspond tout à fait aux représentations sylvestres véhiculées par la Vulgate et par les commentaires patristiques et théologiques.

Mais il importe de bien noter que le référent systématique de cette construction spatiale et temporelle est la chasse : c'est par rapport à cette pratique qu'est définie la profondeur de l'orée et la temporalité que je viens d'évoquer. Le lien étroit entre chasse et sylve est d'ailleurs sanctionné par l'association classique, également présente dans les Weistümer envisagés, à la fois étymologique et allitérative, wald und wild (litt. « sylve et gibier »), homologique à la paire silva/silvester. La forêt est ainsi certes un endroit où il y a des arbres (en fait du bois), mais surtout du gibier - ce qui correspond d'ailleurs bien aux multiples mentions de bêtes, lions, bêtes féroces, sangliers, etc., systématiquement associés à la forêt, dans la Vulgate et les commentaires patristiques ou théologique. Mais ce lien n'est pas seulement un lien de nécessité (le gibier vit en forêt, donc c'est là qu'on le chasse), car la chasse est aussi ce qui permet de souligner la particularité de cet espace.

C'est ce que montre bien la clause 4 stipulant les conditions «étranges » dans lesquelles la chasse au cerf peut être pratiquée : on ne peut chasser le cerf qu'avec un cheval blanc, un braque blanc aux oreilles dressées, un arc en bois d'if à corde de soie, une flèche à fût de plume d'autruche, pointe d'or et empennage de plume d'aigle. La signification de l'insistance sur la couleur du cheval et du chien ne se trouve pas au niveau des couleurs elles-mêmes: elles n'ont aucune nécessité intrinsèque comme l'indique le fait que, dans les versions suivantes, les indications de couleurs changent 37. Ces mesures vont en fait dans le même sens que les spécifications concernant l'arc et la flèche : ces stipulations n'ont aucun sens en soi, elles ne doivent pas être envisagées du point de vue de leur effectivité éventuelle, comme des mesures folkloriques, mais du point de vue de leur effet global.

Toutes ces mesures produisent en effet une logique distinctive à propos de la chasse : ce n'est pas une activité "normale », elle relève d'un espace et d'une logique sociale particuliers. Cette logique distinctive réserve certes implicitement la chasse à l'aristocratie, mais surtout fait de la sylve le lieu [312] de réalisation d'une pratique « extra-ordinaire » - et c'est justement par rapport à elle que le Salzforst est défini comme un ensemble spatio-temporel à part, dans lequel valent d'autres modalités d'organisation du temps et de l'espace que dans le plat pays. La chasse n'est ainsi pas une activité qui se déroule dans la sylve, elle est ce qui la définit comme telle. La sylve n'est pas conçue comme un espace boisé (même s'il y a des $\operatorname{arbres}^{38}$ ) ni comme un

\footnotetext{
37. Le cheval devient ainsi bunt (c'est-à-dire multicolore ou noir et blanc) et le braque sans indication de couleur.

${ }^{38}$. En fait, du bois : comme on l'a déjà signalé, les arbres ne sont évoqués que selon leur essence, et non pas en tant qu'éléments du paysage ; et la clause 14 montre que la sylve peut aussi se composer d'espaces défrichés ( Item ce qui de la forêt a été transformé en champ puis retourne à la forêt doit de nouveau faire partie de la forêt »).
} 
espace animal39, mais comme un espace défini par une pratique sociale construite autour d'un certain type d'animal : la chasse, et tout particulièrement la chasse au cerf.

Les raisons pour lesquelles le gibier principal est le cerf dépassent le seul cadre du Salzforst puisque sa priorité sur tous les autres animaux ${ }^{40}$ se rencontre dans l'ensemble de l'Occident féodal. Or il s'agit d'un animal dont la première caractéristique reconnue est la couardise, ce qui le rend méprisable comme gibier aristocratique dans de nombreuses civilisations, qui l'abandonnent aux pères de famille en quête de viande. Mais au plus tard à partir du XII ${ }^{\mathrm{e}}$ siècle, la chasse au cerf remplace la chasse au sanglier dans la hiérarchie des chasses de l'aristocratie, qui se le réserve tout en en consommant très peu (l'archéoostéologie est formelle là-dessus) : il est donné aux chiens, aux malades, distribué en cadeaux ${ }^{41}$.

Mais un tel paradoxe se résout dès lors que l'on abandonne notre image actuelle de la chasse, celle du sens commun ${ }^{42}$, et que l'on considère la chasse aristocratique médiévale non comme une entreprise d'abattage [313] d'animaux, mais comme une poursuite, une quête (ce qui est congruent avec le sens global de jagen et les transferts métaphoriques de la chasse sur l'amour courtois ou la quête de Dieu43). Si l'on se rappelle aussi l'association wald/wild/wege et si l'on ne réduit pas cette association à un simple jeu de sons ni à une simple évidence pratique (il y a des chemins dans la forêt...) puisqu'il y a bien d'autres choses qui auraient pu être évoquées (par exemple les rivières), alors on pourra admettre que le principe actif de la chasse consiste dans la déambulation dans la sylve à la poursuite d'animaux. Et c'est parce que la chasse aristocratique est une déambulation que l'objet de celle-ci devait logiquement être le plus fuyard (et résistant) des animaux, dont le trajet imprévisible et « brownien » et sa poursuite par un équipage sonore permettaient d'occuper l'espace sylvestre - et de lui donner ainsi une consistance...

Les effets de la déambulation sylvestre des chasseurs sont doubles. D’une part, la chasse permet l'appropriation seigneuriale de l'espace forestier. Ceci attire notre attention sur le fait qu'en l'absence de tout système cadastral, de toute notion correspondant à la " propriété » au sens romain (et actuel) du terme et même de toute conception de l'espace en tant qu'étendue en deux dimensions numériquement mesurables (d'où l'absence de cartographie au sens propre), l'appropriation de l'espace ne pouvait se faire que par l'intermédiaire de son usage ${ }^{44}$. Alors que l'espace agricole était approprié à travers le travail paysan et le fait que ce travail était effectué au

39. La clause 13 exclut la présence de troupeaux dans la sylve («Item personne ne doit non plus mener ou faire garder son bétail dans la Salzforst »).

40. Indépendamment des formes de chasse, puisque la chasse à courre (basée sur la poursuite jusqu'à épuisement) n'est pas pratiquée dans l'Empire, où la chasse au cerf prend la forme du pirschjagd (ce qu'évoquent les Weistümer considérés), c'est-à-dire la poursuite et l'encerclement du cerf pour pouvoir le tirer à l'arc ou à l'arbalète (cf. par exemple le tableau de Lucas Cranach intitulé Die Hirschjagd (1540), aujourd'hui au Cleveland Museum of Arts).

41. M. PASTOUREAU, « La chasse au sanglier : histoire d'une dévalorisation (IVe-XIVe siècle) », dans : La chasse au Moyen Âge. Société, traités, symboles, A. PARAVIcIni-BAGLIANI, B. van den AbEELE dir., Florence, 2000, p. 7-24 ; A. GUERREAU, « La chasse comme rite biparti symétrique », ibid., p. 25-32 ; MORSEL, « Jagd und Raum », loc. cit.

42. Je signale cependant que la définition actuelle de la chasse par l'Association allemande de la chasse est " toute manière d'activité par laquelle les hommes s'emparent d'animaux vivants » (jede Art von Betätigung, durch die sich Menschen in den Besitz freilebender Tiere bringen) : la logique de la chasse relèverait donc du binôme liberté/capture et non pas vie/mort. En France, les chasseurs obsédés par l'abattage du gibier sont (dis)qualifiés de « viandards » par les tenants d'une pratique plus « classique » et respectueuse de l'animal.

43. Cf. A. Guerreau, art. "Chasse », op. cit., et A. GuerREAU-JALABERT, « Le cerf et l'épervier dans la structure du prologue d'Érec », dans : La chasse au Moyen Âge..., op.cit., p. 203-219. Une des figures clés de la quête cynégétique de Dieu est celle de la chasse à la licorne, dont on trouve une bonne représentation figurée dans la collégiale d'Erfurt, datant de 1420.

44. Dès lors, des usages concurrents (ceux des ruraux, éventuellement requalifiés en «braconnage ») induisaient non seulement une infraction aux droits exclusifs de l'évêque, ce qui serait une lecture juridiste, mais surtout une appropriation concurrente de la sylve, selon toute vraisemblance dotée d'une spatialité (et d'une temporalité, les deux choses n’étant pas séparables) alternative mais que nous n’avons pas les moyens, me semble-t-il, de connaître. 
nom/au profit de tel seigneur45, l'absence de gens installés dans la sylve et la faible densité des gens qui y allaient imposaient un autre mode d'appropriation : c'est par le biais des animaux sauvages que cela se faisait, d'où l'interdiction de la pâture du bétail46. Corrélativement, cette [314] appropriation par la chasse rendait inutile l'implantation de représentants du seigneur dans la sylve - et l'on a vu qu'elle se réalisait effectivement seulement en périphérie. À travers le contrôle de la chasse, c'est donc en réalité une forme de contrôle de l'espace qui est mise en œuvre47, par la déambulation.

Mais d'autre part, et consécutivement à ce qui vient d'être dit, cette pratique à la fois collective et audible de poursuite d'un animal sauvage est ce qui donne sa consistance à l'espace sylvestre au-delà de ses éléments structuraux, son périmètre et ses chemins : c'est ce qui constitue la forêt proprement dite - et c'est pour cette raison que la chasse est défendue à l'orée du bois. Il ne s'agit pas de protéger les animaux, mais de signaler que l'on sort (ou qu'on est pas encore) dans la sylve, même s'il y a déjà des arbres.

On voit ainsi tout l'intérêt que présentent les Weistümer du Salzforst examinés. Au delà d'une simple définition normatives des droits de l'évêque, ils le construisent comme un espace spécifique : le marquage périphérique et ponctuel par les tenures et le marquage linéaire par la chasse en construisent la spatialité, en-dehors de toute référence à une quelconque notion d'étendue; et des pratiques cynégétiques à première vue aberrantes en font un espace séparé, hors des cadres spatio-temporels valables pour le reste du monde chrétien, ce qui en retour permet d'appréhender certains aspects de la spatialité médiévale.

\footnotetext{
45. C'est ce que j'ai appelé le "principe de Carabas » : MorSEL, «Jagd und Raum », op. cit., p. 282 ; L'aristocratie médiévale. La domination sociale en Occident (Ve- $X V^{e}$ siècles), Paris, 2004, p. 173.

46. L'appropriation (et la qualification) de l'espace par le biais de pratiques animales ne se réduit pas à la chasse en forêt : on se rappellera également le rôle des troupeaux de moutons sur les friches, des colombes sur les terres cultivées, des chevaux (et des chiens) partout. Je n'ai pas eu la possibilité de consulter l'ouvrage récent de F. DUCEPPE-LAMARRE, Chasse et pâturage dans les forêts du Nord de la France. Pour une archéologie du paysage sylvestre (XIe-XVIe siècles), Paris, 2006.

47. De ce fait, il est rigoureusement logique que les sceaux des Montfort, anciens forestiers de la forêt d'Yveline, représentent, pour figurer l'office forestier sur lequel s'est fondée leur puissance, un chasseur, soit sur le sceau (Simon III de Montfort, comte d'Évreux, en 1140), soit sur le contre-sceau (Philippe de Montfort, seigneur de Castres, v. 1230) d'un sceau équestre.
} 


\section{Weistum sur le Salzforst de 1326}

Ditz sint die reht des waldes, der geheizzen ist der Saltzfoerst, die da zu hat myn herre von Wirtzbuerg vnd sin forstmeyster von sinen wegen.

Ez sal kuntlichen sin allen den dy ${ }^{\mathrm{v}} \mathrm{v}^{\mathrm{e}}$ sint oder her noch kunftig werden, das noch gotes geburte drutzehen hundert iar dar nach in dem sehs vnd zwenzigsten iare an dem nehsten tage nach sancte Walpurg tage eyn forstding besezzen wart von dem forstmeyster vnd allen forstmeystern $\mathrm{zu}^{\mathrm{e}}$ der $\mathrm{Nu}^{\mathrm{e}}$ wenstat in gegenwertikeyt hern Iohansen Fuhs von Swanberg, der zu der zit do eyn voyt was. Vnd wuerden von in vf den eyt geteilt dise reht als her nach ist geschriben.

[1] Zv dem ersten so wart geteilt uf den eyt, daz min herre von Wirtzburg vnd sin vorstmeyster von sinen wegen hab gantzen gewalt vnd vollez reht zu dem vorgenanten walde zu allem wilde vnd wegen.

[2] Item ez ist auch geteilt vf den eyt, daz ein grefe von Hennenberg reht habe, drystunt $\mathrm{zu}^{\mathrm{e}}$ iagen : vnd daz ist eyns in der veiste, daz ander in der roete, daz dritte in der bruenft. Vnd mag haben in dem walde eynen butener vnd eynen wegener.

[3] Ez ist auch geurteilt vf den eyt, daz der vogt des selben waldes sol haben eynen kneht. Vnd der sol niht pfenden vmb vermy ${ }^{v}$ tet holtz.

[4] Ez ist auch geurteilt vf den eyt, daz in dem vorgenanten walde nyeman birsen sal, er habe denne eyn wis pfert vnd eynen wizzen bracken mit gesprentzten o ${ }^{e}$ ren, eynen ybinen bogen mit eyner sydinen snuer, eynen strue zsin schaft mit eyner guldin strael geuidert mit gyres vedern.

[5] Ez sol auch ny ${ }^{v}$ man keyn wilt iagen in sinem ingange noch in sinem vzgange des selben waldes in der banmyvle :

$$
\begin{aligned}
& \text { vnd daz ist eyns hornes gescholle, } \\
& \text { eynes hundes gebelle, } \\
& \text { eynes hamers wurf } \\
& \text { vnd eyns schalkes furtz. }
\end{aligned}
$$

[6] Man sal dem luzser die garn vf dem rucke verbrennen, item eynem stricker den rehten dvmen abslahen, item eynem druher den rehten fu $\mathrm{z}$ abslahen.

[7] Ez ist auch geurteilt vf den eyt, daz die burgman vnd die buerger zu der $\mathrm{Nu}^{\mathrm{e}}$ wenstat haben glichez reht zu hauwen kaltez holtz on laube des vorstmeysters.

[316] [8] Item sy mugen niht hauewen bolyn stueben kelre treme vnd keltur, si geben denne dem vorstmeyster sine schenke ${ }^{48}$.

[9] Item ez sol auch keyn forster pfenden in des andern ampte.

[10] Item daz tal Eberspach vnd der andern dorffer, dye da gelegen sint, vnd daz tal Scho'nowe haben reht zu hau ${ }^{\mathrm{e}}$ wen kalt holtz vnd brunne holtz.

[11] Item sy mugen auch hauwen bueholtz zu irem selbis buwe ; sy ensollens abir nyvt verkauffen.

[12] Item die zilhube sollen das selbe reht haben. 48. Dans une version postérieure (1353), les dons sont précisés : 2 gélines, ou 1 quartier de vin, ou 1 pain blanc valant 1
sou heller. 
[13] Item keyn forster sol keyn bete geben von siner forsthuebe. Von andern iren guten sollen sie tuen als ander lute.

[14] Item ez sal auch nyvman sin vihe triben nach lazzen hueten in dem Saltzforste.

[15] Item was von dem walde zu acker gemacht ist vnd wider $\mathrm{z}^{\mathrm{e}} \mathrm{u}$ walde wirt daz sol wyder zu dem walde geho ${ }^{\mathrm{e}} \mathrm{ren}$.

Source: copie contemporaine49 éditée dans Monumenta Boica, t. 39, Munich, 1866, $\mathrm{n}^{\circ}$ CXXXIX, p. 277-279.

49. Dans un cahier de parchemin introduit par la mention Tempore domini Friderici de Roten[ko]lben magistri nemoris. Actum anno domini $M^{\circ} C C C^{\circ} X X V I^{\circ}$ in crastino Walpurgis virginis (26 février 1326). 